\title{
Quality improvement of partially coherent symmetric-intensity beams caused by quartic phase distortions
}

\author{
R. Martínez-Herrero, P. M. Mejías, and G. Piquero \\ Departamento de Optica, Facultad de Ciencias Fisicas, Universidad Complutense, 28040-Madrid, Spain
}

Received July 7, 1992

\begin{abstract}
The effects that quartic phase distortions produce in the beam-quality parameter of partially coherent symmetric-intensity beams are studied. An analytical expression for the beam-quality parameter at the output plane of a pure phase plate with quartic phase aberration has been derived. Explicit conditions to improve the beam quality are provided, and the corresponding optimized beam-quality value that can be attained for a given field has been determined.
\end{abstract}

The characterization of the spatial profiles of laser beams and the analysis of their quality changes under propagation through general optical systems are topics of increasing interest in the literature. ${ }^{1-9}$ One of the major problems is to improve the beam quality, usually defined in terms of the so-called moments of both the intensity and the radiant intensity of the field. In this sense, use of pure phase transmittances through which the beam is made to propagate seems to be an appropriate procedure because the total beam power is maintained.

In this Letter quartic phase distortions are considered and an analytical formula is derived for the changes introduced in the beam-quality parameter of partially coherent symmetric-intensity beams (note that the Hermite-Gauss laser-beam modes belong to this type of symmetric fields). But this class of phase transmittance function could also represent thermal effects generated by high-power lasers or even spherical aberration in lenses. Accordingly, our results also apply to describe these situations. Explicit conditions to improve the beam quality are given, and the optimized quality value that it is possible to attain for a fixed beam with this kind of pure phase plate is found. From the above results we show the rather surprising conclusion that, in certain cases, a given degree of spherical aberration could improve the beam quality. Our results agree with those obtained by Siegman ${ }^{10}$ in the particular case of coherent laser beams with quadratic phase terms degraded by quartic phase aberrations.

Let us then start from the cross-spectral density (CSD) function of a partially coherent symmetricintensity beam written in the form

$$
G\left(x_{1}, x_{2}\right), \quad G(x, x)=G(-x,-x)=I(x),
$$

where $x, x_{1}$, and $x_{2}$ are variables transverse to the propagation direction $z$ (for simplicity, we consider the bidimensional case) and $I(x)$ represents the beam intensity. It is clear that the CSD function at the output of a pure phase plate whose transmittance is given by the function $\exp [i k f(x)], k$ being the wave number of the field, becomes

$$
G_{o}\left(x_{1}, x_{2}\right)=G_{i}\left(x_{1}, x_{2}\right) \exp \left\{i k\left[f\left(x_{2}\right)-f\left(x_{1}\right)\right]\right\},
$$

where the subscripts $o$ and $i$ denote the values at the output and input planes, respectively, of the phase plate. We are considering quasimonochromatic fields, so explicit dependence on frequency is suppressed in our expressions. Let us now introduce the Wigner distribution function $h$ associated with the CSD in the usual form:

$$
\begin{aligned}
& h(x, u, z) \\
& \quad=(1 / H) \int G(x+s / 2, x-s / 2, z) \exp (-i k u s) \mathrm{d} s,
\end{aligned}
$$

where

$$
H=\int h \mathrm{~d} x \mathrm{~d} u
$$

is a normalization constant (division by the total power is implicit) and $k u$ provides the wave vector component along $x$ (hence $u$ represents an angle of propagation, without taking the evanescent waves into account). It is well known that integration of $h$ over the angular variable $u$ gives the beam intensity, and, on the other hand, the integral over the spatial variable $x$ provides the directional intensity, which is, adding the factor $\cos ^{2} \beta$ ( $\beta$ being the angle of observation), proportional to the radiant intensity of the field. Averages (moments) of the Wigner distribution function can be defined as follows:

$$
\left\langle x^{m} u^{n}\right\rangle=\int x^{m} u^{n} h(x, u, z) \mathrm{d} x \mathrm{~d} u .
$$

As is well known, first and second-order moments $\langle x\rangle,\langle u\rangle,\left\langle x^{2}\right\rangle,\left\langle u^{2}\right\rangle$, and $\langle x u\rangle$ are closely connected, respectively, with the mean position of the beam, the mean propagation direction of the beam, the beam width, the far-field divergence, and the inverse of the effective radius of curvature at the plane that we are considering. From Eqs. (3)-(5), after lengthy but straightforward calculations, it can be shown that 


$$
\begin{aligned}
\langle x\rangle_{o} & =\langle x\rangle_{i}=0 \\
\left\langle x^{2}\right\rangle_{o} & =\left\langle x^{2}\right\rangle_{i} \\
\langle u\rangle_{o} & =\langle u\rangle_{i}+(1 / P) \int f^{\prime}(x) G_{i}(x, x) \mathrm{d} x \\
u\rangle_{o} & =\langle x u\rangle_{i}+(1 / P) \int x f^{\prime}(x) G_{i}(x, x) \mathrm{d} x \\
\left\langle u^{2}\right\rangle_{o}=\left\langle u^{2}\right\rangle_{i}+(i / k P) \int f^{\prime}(x)\left\{\left[\partial G_{i}\left(x_{1}, x_{2}\right) / \partial x_{1}\right]_{x_{1}=x_{2}=x}\right. & \\
- & {\left.\left[\partial G_{i}\left(x_{1}, x_{2}\right) / \partial x_{2}\right]_{x_{1}=x_{2}=x}\right\} \mathrm{d} x } \\
+ & (1 / P) \int\left[f^{\prime}(x)\right]^{2} G_{i}(x, x) \mathrm{d} x
\end{aligned}
$$

along with $I_{o}(x)=I_{i}(x)$, where $P=\int G(x, x) \mathrm{d} x$ and the prime indicates derivation with respect to $x$. Equations (6) -(10) apply for arbitrary transmittance functions $f(x)$. But here we are interested in quartic phase distortions, i.e.,

$$
f(x)=a x^{2}+b x^{4} .
$$

Substitution of Eq. (11) into Eqs. (6)-(10) gives

$$
\begin{gathered}
\langle u\rangle_{o}=0, \\
\left(\langle x u\rangle_{o}\right)^{2}=\left(\langle x u\rangle_{i}\right)^{2}+4 a^{2}\left(\left\langle x^{2}\right\rangle_{i}\right)^{2} \\
+16 b^{2}\left(\left\langle x^{4}\right\rangle_{i}\right)^{2}+4 a\langle x u\rangle_{i}\left\langle x^{2}\right\rangle_{i} \\
+8 b\left\langle x^{4}\right\rangle_{i}\langle x u\rangle_{i}+16 a b\left\langle x^{4}\right\rangle_{i}\left\langle x^{2}\right\rangle_{i}, \\
\left\langle u^{2}\right\rangle_{o}=\left\langle u^{2}\right\rangle_{i}+4 a\langle x u\rangle_{i}+8 b\left\langle x^{3} u\right\rangle_{i} \\
+4 a^{2}\left\langle x^{2}\right\rangle_{i}+16 b^{2}\left\langle x^{6}\right\rangle_{i}+16 a b\left\langle x^{4}\right\rangle_{i},
\end{gathered}
$$

where it has been assumed, for the sake of simplicity, that $\langle u\rangle_{i}=0$ (otherwise, it will suffice to change the propagation direction to meet such a requirement). It is important to notice that the values of the secondorder beam moments at the output of a quartic phase plate depend on the values of higher-order beam moments at the input plane.

Let us now introduce the beam-quality parameter defined in the form (when $\langle x\rangle=\langle u\rangle=0)^{7-9}$

$$
Q=\left\langle x^{2}\right\rangle\left\langle u^{2}\right\rangle-\langle x u\rangle^{2} .
$$

In the literature, it is also usual to handle another quality parameter, namely the so-called $M^{2}$ parameter, ${ }^{4}$ closely related to $Q$ by means of the equation

$$
Q=\left(M^{2}\right)^{2} / 4 k^{2},
$$

with $M^{2} \geq 1$. From Eq. (15), it can immediately be shown that

$$
Q_{o}-Q_{i}=\Delta Q=16 b^{2} S+8 b T,
$$

where $\Delta Q$ is called the quality gain factor and

$$
\begin{aligned}
& S=\left\langle x^{6}\right\rangle_{i}\left\langle x^{2}\right\rangle_{i}-\left(\left\langle x^{4}\right\rangle_{i}\right)^{2}, \\
& T=\left\langle x^{3} u\right\rangle_{i}\left\langle x^{2}\right\rangle_{i}-\left\langle x^{4}\right\rangle_{i}\langle x u\rangle_{i} .
\end{aligned}
$$

A number of interesting properties can be inferred from the above equations:

(1) When $T=0$, the quality gain factor is positive. In other words, the quality is reduced.

(2) When $T \neq 0$, the quality gain factor is negative (increased quality) if

$$
\begin{aligned}
& \text { (i) } \operatorname{sgn}(b) \neq \operatorname{sgn}(T) \text {, } \\
& \text { (ii) }|b|<|T| / 2 S \text {. }
\end{aligned}
$$

(3) The beam quality is optimized when

$$
|b|=|T| / 4 S \text {. }
$$

Property (1) follows from the fact that $S>0$. Property (2) can be shown by direct substitution of conditions (20) and (21) into Eq. (17) in the two possible cases ( $T>0$ or $T<0$ ). Property (3) follows by taking into account that

$$
\partial^{2}(\Delta Q) / \partial b^{2}>0,
$$

when

$$
b=-[\operatorname{sgn}(T)]|T| / 4 S .
$$

As a final point of interest we have that, for those beams whose CSD functions are of the form

$$
G\left(x_{1}, x_{2}\right)=A\left(x_{1}, x_{2}\right) \exp \left[i k d\left(x_{2}{ }^{2}-x_{1}^{2}\right)\right],
$$

where $A$ takes real values and $d$ is a constant also real, the quality is always reduced. This can readily be shown by noting that, for this class of fields, $T=0$. Important examples of this type of beam for which quartic phase distortions are of no use at all to improve their quality are Gauss-Schell model fields and Hermite-Gauss laser beams. This final property agrees with the result obtained by Siegman, ${ }^{10}$ who demonstrated that quartic phase aberrations always degrade the quality factor of coherent laser beams with quadratic phase terms.

We acknowledge the financial support of the Comisión Interministerial de Ciencia y Tecnología of Spain under Project ROB90-53.

\section{References}

1. M. J. Bastiaans, J. Opt. Soc. Am. A 3, 1227 (1986).

2. S. Lavi, R. Prochaska, and E. Keren, Appl. Opt. 27, 3696 (1988).

3. R. Simon, N. Mukunda, and E. C. G. Sudarshan, Opt. Commun. 65, 322 (1988).

4. A. E. Siegman, Proc. Soc. Photo-Opt. Instrum. Eng. 1224, 2 (1990).

5. P. A. Bélanger, Opt. Lett. 16, 196 (1991).

6. A. E. Siegman, IEEE J. Quantum Electron. 27, 1146 (1991).

7. J. Serna, R. Martínez-Herrero, and P. M. Mejías, J. Opt. Soc. Am. A 8, 1094 (1991).

8. R. Martínez-Herrero and P. M. Mejías, Opt. Commun. 85, 162 (1991).

9. J. Serna, P. M. Mejías, and R. Martínez-Herrero, J. Mod. Opt. 39, 625 (1992).

10. A. E. Siegman, "Analysis of laser beam quality degradation cause by quartic phase aberrations," submitted to Appl. Opt. 